\title{
Effect of age and pregnancy status on adaptive immune responses of Canadian Holstein replacement heifers
}

\author{
B. C. Hine, ${ }^{1}$ S. L. Cartwright, and B. A. Mallard \\ Department of Pathobiology, Ontario Veterinary College, University of Guelph, ON, Canada N1G 2W1
}

\begin{abstract}
Selection for production traits with little or no emphasis on health traits has led to an increase in the incidence of disease in Canadian dairy herds. We describe here a patented protocol for estimating the breeding value for immune responsiveness in heifers that combines measures of both cell-mediated (CM) and antibody-mediated (AM) immune responses (IR). The ability of putative type 1 and type 2 antigens used to induce CMIR and AMIR, respectively, was assessed in replacement Holstein heifers, and the effects of age and pregnancy on type 1 and type 2 IR bias were estimated. Results demonstrated that the type 1 and type 2 antigens induced polarized type 1 and type 2 responses in heifers regardless of age and pregnancy status, and can therefore be used to identify animals with superior overall immune responsiveness. However, age and pregnancy status had significant effects on adaptive IR profiles, highlighting the need for appropriate statistical modeling of such effects when ranking animals on their ability to mount CM and AMIR. Responses became increasingly type 1 biased as heifers approached 12 mo of age, from which point, responses then became increasingly type 2 biased with age and length of gestation. Knowledge of how age and pregnancy influence the dynamics of type 1 and type 2 IR bias is expected to improve our ability to select animals with enhanced immune responsiveness and aid in the development of effective vaccines through strategic targeting of vaccine components to recipients.
\end{abstract}

Key words: dairy heifer, immune response, selection, type 1 type 2 immune response bias

\section{INTRODUCTION}

Antagonistic genetic correlations between milk production and the incidence of many common diseases of dairy cattle suggest that disease incidence will increase in herds where selection is largely based on production

Received August 8, 2010.

Accepted October 21, 2010.

${ }^{1}$ Corresponding author: brad.hine@agresearch.co.nz traits with little or no emphasis on health traits (Lyons et al., 1991; Van Dorp et al., 1998). For example, the genetic correlation between milk production and the incidence of mastitis has been estimated at between 0.15 and 0.37 (Lyons et al., 1991; Uribe et al., 1995; Van Dorp et al., 1998). A possible genetic solution to this problem is to combine production and immune response (IR) traits into a weighted selection index with the aim of breeding high-producing animals with enhanced overall immune responsiveness (Mallard et al., 1998b; Wilkie and Mallard, 1999).

A patented protocol (Mallard and Wagter, 2001) has been developed and tested to estimate the breeding value of adaptive immune responsiveness of individual animals as measured by their ability to mount both cell-mediated $(\mathbf{C M})$ and antibody-mediated (AM) IR (Mallard et al., 1998b; Hernández et al., 2003; Heriazon et al., 2009b). Animals generally respond to intracellular pathogens by mounting a CMIR and to extracellular pathogens and soluble antigens by mounting an AMIR. Cell-mediated and antibody-mediated IR are negatively genetically correlated in dairy cattle (Hernández et al., 2006), suggesting that the selection of cattle based on their resistance to extracellular pathogens may lead to an increase in their susceptibility to intracellular pathogens and vice versa. Selection of animals with a balanced ability to mount both a CMIR and AMIR is expected to lead to improved broad-based disease resistance, improved animal welfare, and a reduced reliance on the use of antibiotics and other therapeutic agents to control infectious disease in the dairy industry.

To date, this IR-testing method has been successfully employed to classify young calves (2-6 mo of age) and mature cows (2 yr of age and older) as high, average, or low immune responders (Nino-Soto et al., 2008; Begley et al., 2009a,b; Heriazon et al., 2009b). However, use of the IR-testing protocol to classify replacement heifers during the period from postweaning through mating and into mid pregnancy has not been investigated. For IR testing to be incorporated into a selection index, it is important that the testing protocol can be successfully used to classify animals of all ages and physiological conditions. Testing of replacement dairy heifers is expected to allow producers to identify and cull those 
animals classified as having a low IR phenotype early in life, minimizing feed and labor input costs. Further, if EBV for IR are to be calculated for sires and made available to producers, IR phenotype testing of a sire's progeny early in life will reduce the time required for sires to be proven, thereby improving genetic gain for enhanced IR.

To measure IR, the testing protocol described here incorporates putative type 1 and type 2 antigens. Type 1 and type 2 antigens preferentially induce production of type 1 (IFN- $\gamma$, IL-12) and type 2 (IL-4, IL-10, IL13) cytokines that direct immune response polarization toward a CMIR or AMIR, respectively (Crawley and Wilkie, 2003). Production of type 2 cytokines also induces eosinophilia and mast cell proliferation. When predicting an animal's overall immune responsiveness, it is important that the test antigens provide an accurate and repeatable estimate of an animal's ability to mount a balanced immune response. The hypothesis tested here was that the putative type 1 and type 2 antigens used in the testing protocol would successfully induce measurable CMIR and AMIR, respectively, in replacement heifers of various ages and physiological states. Further, although age and pregnancy status may have a significant influence on IR, statistical modeling of such effects is expected to permit accurate predictions of overall immune responsiveness and allow for the identification of superior phenotypes. To test this hypothesis, IR testing was conducted on 128 Holstein replacement heifers ranging in age and pregnancy status. The ability of test antigens to induce type 1 and type 2 responses also was assessed, and responses were used to estimate the effects of age and pregnancy status on the adaptive IR profile. A subset of animals identified as having HiAMIR/LoCMIR or LoAMIR/HiCMIR phenotypes underwent a second round of IR testing to assess the stability of phenotypes.

\section{MATERIALS AND METHODS}

\section{Animals}

Primary IR testing was conducted on 128 Canadian Holstein replacement heifers from the University of Guelph's Elora Dairy research herd. Heifers ranged in age from 2 to $26 \mathrm{mo}$, with their ages well distributed over the entire range $(2-6 \mathrm{mo}, \mathrm{n}=30 ; 7-12 \mathrm{mo}, \mathrm{n}$ $=31 ; 12-17 \mathrm{mo}, \mathrm{n}=33$; and $18-25 \mathrm{mo}, \mathrm{n}=34$ ). Of the heifers tested, 85 were not pregnant (NP), 20 were in early pregnancy $(\mathbf{E P}$, gestation $<100 \mathrm{~d})$, and 23 were in mid pregnancy (MP, gestation $=100-200 \mathrm{~d}$ ). Heifers in late pregnancy (gestation $>200 \mathrm{~d}$ ) were not tested as the effects of approaching parturition on IR have been well described (Saad et al., 1989; Nagahata et al., 1992). To assess phenotype stability, a subset of 40 heifers identified as having a HiAMIR/LoCMIR $(\mathrm{n}=20)$ or LoAMIR/HiCMIR $(\mathrm{n}=20)$ phenotype underwent secondary IR testing with the same type 1 and 2 antigens. These phenotypes were selected as they represent relatively common phenotypes in the population. During testing, heifers were housed indoors in group pens and were fed a standard dry cow ration. All experimental procedures were approved by the Animal Care Committee of the University of Guelph under guidelines of the Canadian Council of Animal Care.

\section{Immunization and Blood Sampling}

For logistical reasons, heifers were randomly assigned to 4 sampling groups for primary and secondary IR testing. Several combinations of type 1 and type 2 antigens have been used previously by our group to assess CM and AMIR in cattle (Hernández et al., 2005; Heriazon et al., 2009a,b). Evaluation of various antigen combinations for use in IR testing is ongoing. In the current study, Candida albicans and hen egg white lysozyme (HEWL) were used in IR testing to induce type 1 and type 2 immune responses, respectively. Heifers were immunized on d 0 (the beginning of testing) with $1.0 \mathrm{~mL}$ of sterile PBS solution containing $0.5 \mathrm{mg}$ of the type 1 antigen, killed C. albicans (crude whole cell; Greer Laboratories Inc., Lenoir, NC), $0.5 \mathrm{mg}$ of the type 2 antigen, HEWL (Sigma-Aldrich Canada Ltd., Ontario, Canada), and $0.5 \mathrm{mg}$ of Quil-A adjuvant (Brenntag, Frederikssund, Denmark). Immunizations were given i.m. in the prescapular region. Secondary IR testing was conducted between 91 and $128 \mathrm{~d}$ after primary IR testing (depending on sampling group).

For primary IR testing, blood samples were collected preimmunization (d0P) and $14 \mathrm{~d}$ postimmunization $(\mathbf{d} 14 \mathbf{P})$. For secondary IR testing, blood was collected preimmunization (d0S) and $7 \mathrm{~d}$ postimmunization (d7S). Blood samples were collected via caudal venipuncture, and serum was collected from coagulated blood by centrifugation $(700 \times g, 20 \mathrm{~min})$ and stored at $-20^{\circ} \mathrm{C}$ for subsequent ELISA procedures.

\section{Delayed-Type Hypersensitivity}

To assess CMIR, delayed-type hypersensitivity (DTH) reactions were induced by intradermal (ID) injection of the type 1 antigen into the tail skin-fold on d14P and d7S (subset of animals) as described by Hernández et al. (2005) and Heriazon et al. (2009b). Briefly, $0.1 \mathrm{~mL}$ of sterile PBS solution containing 0.1 mg of the type 1 antigen, killed C. albicans (crude whole cell) (test), and $0.1 \mathrm{~mL}$ of sterile PBS (control) was injected ID into the skin fold on opposite sides of the 
tail. As an indicator of DTH, double skin-fold thickness (DSFT) measurements (3 repetitions) were taken before ID injection (T0) and $48 \mathrm{~h}$ after ID injection (T48) at both test and control sites using a Harpenden spring-loaded dial-caliper (Creative Health Products Inc., Ann Arbor, MI) and recorded in millimeters. For each individual, an increase in DSFT of $>1 \mathrm{~mm}$ at the test site relative to the control site at T48 was considered a positive DTH response.

\section{Serum Antibody}

To assess AMIR, total serum IgG antibody against the type 2 antigen was determined using the indirect ELISA method described by Mallard et al. (1997) with the following minor modifications. Briefly, plates were coated with HEWL. Plates were blocked with a PBS $+3 \%$ polysorbate 20 solution containing $1.5 \%$ BSA and $1.5 \%$ fetal calf serum. All test and control samples were assayed in quadruplicate. Pooled serum samples from $\mathrm{d} 0 \mathrm{P}$ and $\mathrm{d} 14 \mathrm{P}$ were used as negative and positive controls, respectively. For each individual and each IR test, pre- (d0P or dOS) and corresponding postimmunization (d14P or d7S) samples were run in replicates on a single plate. Mean optical density (OD) values for replicates were corrected based on the mean OD value of a positive serum control assayed on the same plate (Mallard et al., 1997). Antigen-specific total IgG was detected using affinity purified sheep antibovine IgG (H-chain specific) conjugated to alkaline phosphatase (AP; product no. A10-118AP, Bethyl Laboratories, Montgomery, TX). For each individual, an increase of $>0.1$ OD units from pre- to postimmunization was considered a positive antibody response.

The ratio of IgG1 to IgG2-related antibodies produced in IR indicates type 1 or type 2 IR bias (Crawley et al., 2005). Therefore, to assess type 1 or type 2 IR bias, isotype-specific IgG1 and IgG2 antibodies against both the type 1 and type 2 antigens also were determined at d0P and $\mathrm{d} 14 \mathrm{P}$ using the indirect ELISA method described above. To determine antibody activity against the type 1 and type 2 antigens, plates were coated with candin, a purified protein from C. albicans (Greer Laboratories Inc., Lenoir, NC) or HEWL, respectively. Antigen-specific IgG1 and IgG2 was detected using affinity purified sheep antibovine IgG1 conjugated to AP (product no. A10-116AP, Bethyl Laboratories) and sheep antibovine IgG2 conjugated to AP (product no. A10-117AP, Bethyl Laboratories), respectively. Due to the low serum IgG2 antibody to the type 2 antigen observed in the current study at $\mathrm{d} 14 \mathrm{P}$, a pooled positive serum control for IgG2 ELISA procedures was sourced from a previous experiment (Heriazon et al., 2009b).

\section{Statistical Analyses}

Antibody activity, IgG-related antibody isotype ratio, and DTH data were analyzed independently with a linear mixed model using Proc MIXED (SAS 9.1.3, Cary, NC). The model was as follows:

$$
\mathbf{y}=\mathbf{X} \boldsymbol{\beta}+\mathbf{Z}_{\gamma}+\varepsilon,
$$

where $\mathbf{y}$ is the response vector for the observations, $\mathbf{X}$ is the model matrix for the fixed effects (pregnancy status, phenotype classification, and the covariate of age [linear and quadratic]), $\boldsymbol{\beta}$ is the vector of fixedeffect coefficients, $\mathbf{Z}$ is the model matrix for the random effects (sampling group), $\boldsymbol{\gamma}$ is the vector of random effects coefficients, and $\varepsilon$ is the vector of errors for the observations.

The effects of age (defined as age in months at d0P or d0S), pregnancy status (NP, EP, or MP), and their interactions were fitted to all models. For analysis of responses to secondary IR testing, the fixed effect of phenotype classification (HiAMIR or HiCMIR, as discussed below) and interactions of phenotype classification also were fitted. The random effect of sampling group (groups 1 to 4) also was fitted to all models. Where baseline preimmunization antibody responses to the type 1 or type 2 antigen were detectable in the majority of animals at testing commencement (d0P or d0S), baseline values were fitted as covariates to models (including interactions), allowing interactions between baseline responses and other effects, such as age and pregnancy status, to be estimated. Where baseline responses were at (or around) the detection limit of the ELISA assay for most animals at the commencement of primary IR testing $(\mathrm{d} 0 \mathrm{P})$, baseline values were subtracted from their corresponding $\mathrm{d} 14 \mathrm{P}$ values.

A split-plot model, in which time (d0P, d14P or d0S, $\mathrm{d} 7 \mathrm{~S}$ ) was included as a factor and cow fitted as a random effect, was used to assess the ability of this immunization protocol to induce AMIR and CMIR. The main effects of age, pregnancy status, and phenotype classification along with relevant covariates were retained in models regardless of their $P$-values. However, models were reduced by removing nonsignificant $(P>0.1)$ interaction terms. When removing nonsignificant interaction terms, hierarchy was preserved at all times and effects involved in significant interactions were retained regardless of their $P$-values. Contrasts were evaluated by Bonferroni $t$ statistics for multiple comparisons.

To test the assumptions of the ANOVA, comprehensive residual analyses were conducted. The PROC UNIVARIATE (SAS 9.1.3) was used to test the residuals for normality (Anderson-Darling, Cramér-von 
Mises, Kolmogorov-Smirnov, and Shapiro-Wilk tests). The residuals were plotted against predicted and explanatory variable values to reveal potential outliers or other problems in the data, and to assess the need for data transformation. An unbiased correction factor was added to response variables where required for transformation. A $P$-value of less than 0.05 was considered significant for reporting purposes.

Unless stated, data for graphical representation of results were generated using statistical model solutions describing predicted effects of model terms. Where significant age $\times$ pregnancy status interactions were observed, data were modeled over biologically relevant age periods $(\mathrm{NP}=2-17 \mathrm{mo}, \mathrm{EP}=14-21 \mathrm{mo}, \mathrm{MP}=$ 17-26 mo). Least squares means (LSM) differences described in the Results section are presented on the log (natural logarithm) scale.

Antibody-Mediated IR. Production of total IgG specific to the type 2 antigen was used as an indicator of AMIR. To estimate effects of age and pregnancy status on AMIR following primary IR testing, total IgG production was calculated by subtracting the log (natural logarithm) of the mean ELISA OD value at d0P from the log of the mean ELISA OD value at d14P and tested as the response variable. To estimate effects of phenotype classification on AMIR following secondary IR testing, total IgG production was calculated by taking the log of the mean ELISA OD value at $\mathrm{d} 7 \mathrm{~S}$ and tested as the response variable with $\log$ of the mean ELISA OD value at d0S fitted as a covariate in the model.

Cell-Mediated IR. Responses to DTH testing were used as an indicator of CMIR. To estimate effects of age and pregnancy status on CMIR following primary IR testing, the log of (DSFT at test site/DSFT at control site) at T48 was tested as the response variable with the log of (DSFT at test site/DSFT at control site) at T0 fitted as a covariate in the model.

Immune Response Bias. The relative production of IgG1 versus IgG2 specific to each test antigen was used as an indicator of IR bias. To estimate effects of age and pregnancy status on IR bias following primary IR testing, IgG1 and IgG2 production specific to the type 1 and type 2 antigens was assessed. For the type 2 antigen, the log of the mean IgGi (where $\mathrm{i}=1$ or 2 ) ELISA OD value at d0P was subtracted from the log of the mean IgGi ELISA OD values at d14P and tested as the response variable. For the type 1 antigen, the log of the mean IgGi ELISA OD value at d14P was tested as the response variable with log of the mean IgGi ELISA OD value at d0P fitted as a covariate in the model.

A similar model was used to analyze the IgG1:IgG2 antibody ratios specific to the type 1 and type 2 antigens. For the type 2 antigen, the log of (the mean IgG1
ELISA OD value/the mean IgG2 ELISA OD value) at $\mathrm{d} 14 \mathrm{P}$ was tested as the response variable. For the type 1 antigen, the log of (the mean IgG1 ELISA OD value/ the mean IgG2 ELISA OD value) at d14P was tested as the response variable, and the log of (the mean IgG1 ELISA OD/ the mean IgG2 ELISA OD) at d0P fitted as a covariate in the model.

Phenotypic Classification of Animals for Secondary Immune Response Testing. To identify HiAMIR/LoCMIR and LoAMIR/HiCMIR phenotypes, heifers were ranked based on model residual (observed - predicted) values. Residuals for ranking on CMIR and AMIR were generated from the models described above. In an effort to balance age and pregnancy status within phenotype classifications, 5 heifers from each of 4 age categories $(2-7,7-11,11-16$, and 16-26 mo of age) were identified as having a HiAMIR/LoCMIR phenotype, and a further 5 from each age category were identified as having a LoAMIR/HiCMIR phenotype. Those individuals classified as having a HiAMIR/LoCMIR phenotype had the largest positive AMIR residual value within their age category while also having a negative CMIR residual value (Figure 1). In contrast, those individuals classified as having a LoAMIR/HiCMIR phenotype had the largest positive CMIR residual value within their age category while also having a negative AMIR residual value.

\section{RESULTS}

\section{Responses to Primary IR Testing}

Primary immunization led to an increase in total IgG antibody to the type 2 antigen at $\mathrm{d} 14 \mathrm{P}$ relative to d0P (LSM difference $=1.296 \pm 0.097, P<0.001$ ). Positive antibody responses to the type 2 antigen were observed in $82 \%$ of heifers that underwent primary IR testing. The age and pregnancy status of the heifers, which did not respond to primary immunization were varied, suggesting that nonresponsiveness to the type 2 antigen was not localized to a specific age category or physiological state. Positive DTH responses to the type 1 antigen were observed in $99 \%$ of heifers that underwent primary IR testing. Primary DTH testing resulted in an increase in DSFT measurements at the test site at T48 compared with T0 (LSM difference $=$ $0.714 \pm 0.036, P<0.001)$.

\section{Effects of Age and Pregnancy Status on Responses to Primary IR Testing}

Antibody-Mediated IR. Heifer age $(P=0.034)$ but not pregnancy status $(P=0.670)$ influenced AMIR induced by primary immunization (Table 1). Results 


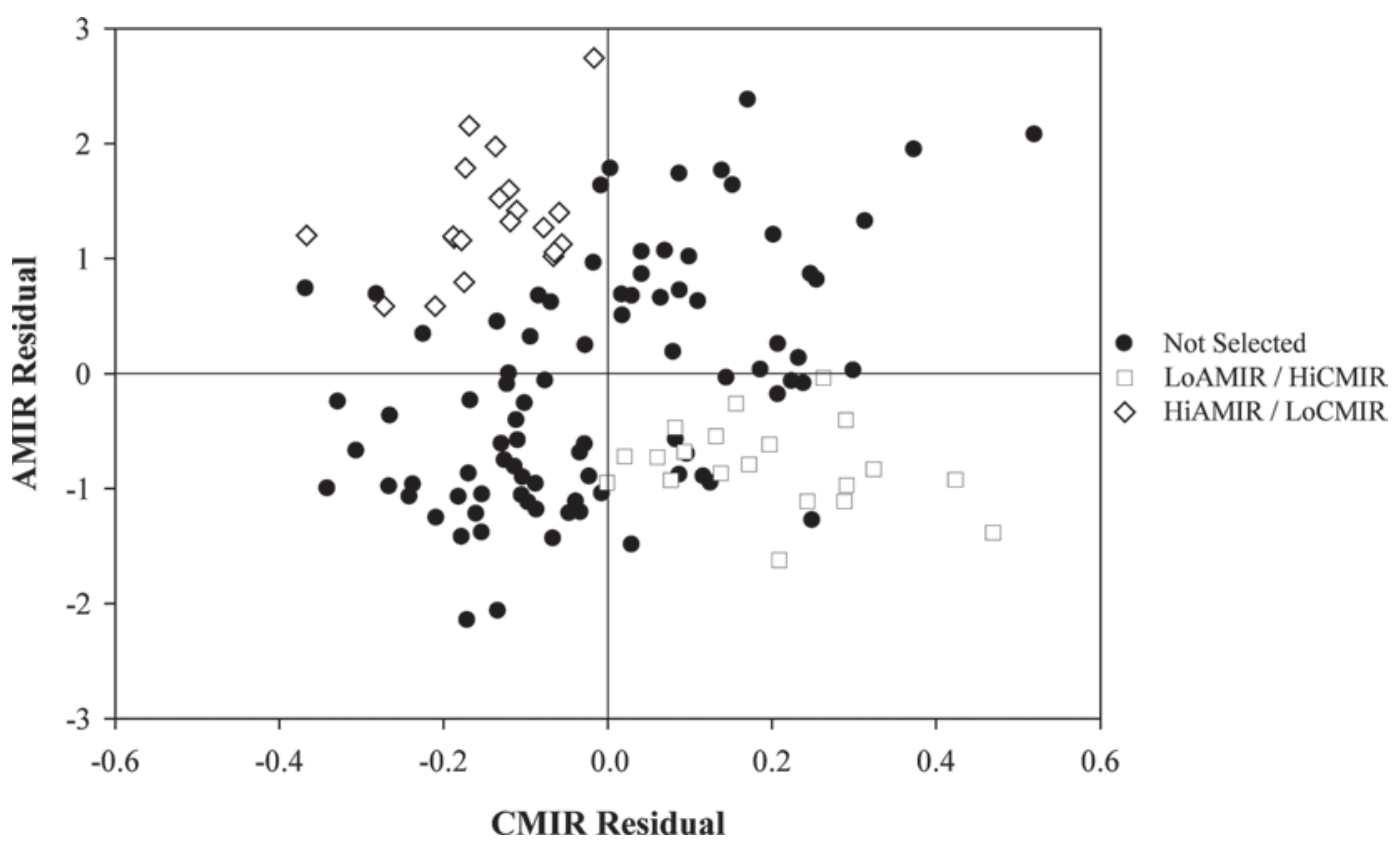

Figure 1. Residual plot depicting heifers classified as low (Lo)AMIR/high (Hi)CMIR or HiAMIR/LoCMIR phenotypes that underwent secondary immune response testing in which antibody-mediated (AMIR) and cell-mediated immune response (CMIR) are defined as total IgG antibody to the type 2 antigen and delayed-type hypersensitivity reactions to the type 1 antigen, respectively.

indicate that an animal's ability to mount AMIR, as measured by production of IgG against the type 2 antigen, is high in young calves $(2-3 \mathrm{mo})$, steadily declines as they approach 12 mo of age and then increases continually with age to peak at 25-26 mo, the oldest age group tested (Figure 2A).
DTH. Age influenced CMIR; however, the effect varied depending on pregnancy status (age $\times$ status, $P<0.001$; age $\times$ age $\times$ status, $P<0.001)$ (Table 1 ). The ability of calves to mount a CMIR was lowest in young calves $(2-3 \mathrm{mo})$, increased until heifers reached approximately 12 mo of age, and then steadily declined

Table 1. Probabilities for the effects of age and pregnancy on primary and secondary immune responses in Holstein heifers ${ }^{1}$

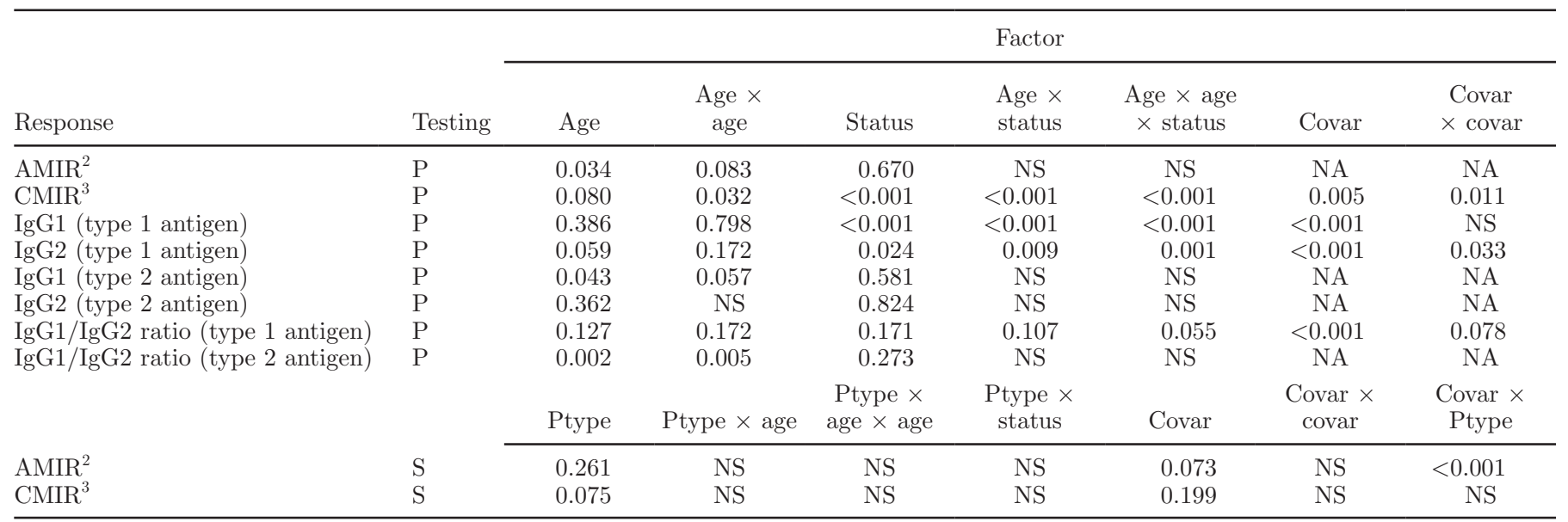

${ }^{1}$ Testing: $\mathrm{P}=$ primary immune response testing; $\mathrm{S}=$ secondary immune response testing; Status = pregnancy status; Covar = covariate (baseline value measured at beginning of testing, d 0); Ptype $=$ phenotype classification. Numbers in the table represent $P$-values $($ ANOVA) describing the effect of each factor on the measured response. NS $=$ factors removed from model statement $(P>0.1)$. NA $=$ factor not fitted.

${ }^{2} \mathrm{AMIR}=$ antibody-mediated immune response as defined by total IgG antibody activity against the type 2 antigen used in immune response testing.

${ }^{3} \mathrm{CMIR}=$ cell-mediated immune response as defined by delayed type hypersensitivity reaction against the type 1 antigen used in immune response testing. 

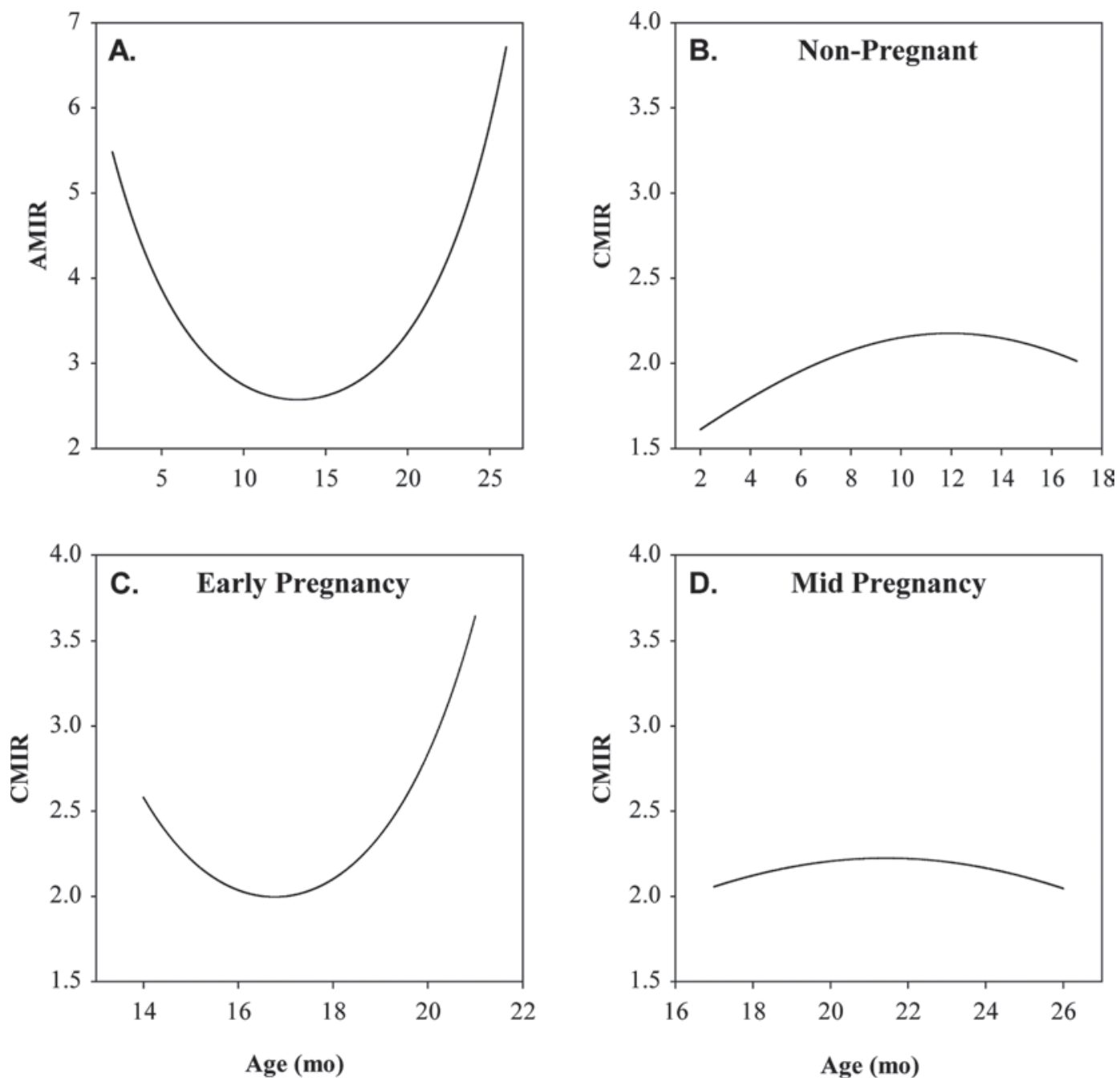

Figure 2. Effect of age on AMIR (A) and CMIR during the not pregnant (B), early pregnancy (C), and mid pregnancy (D) periods, where antibody-mediated (AMIR) and cell-mediated (CMIR) immune response are defined as total IgG antibody to the type 2 antigen and delayedtype hypersensitivity reactions to the type 1 antigen, respectively. For CMIR (but not AMIR) the effect of age varied significantly with pregnancy status.

again with age (Figure 2B). The most dramatic effect of age was observed during EP, when ability to mount a CMIR increased with age (and associated gestation length) from around 17 mo of age (Figure 2C).

\section{Type 1/Type 2 Immune Response Bias}

Heifer age (age $P=0.002$, age $\times$ age $P=0.005$ ), but not pregnancy status $(P=0.273)$, had an effect on the IgG1:IgG2 antibody ratio toward the type 2 antigen (Table 1, Figure 3A) and although no significant effects of age or pregnancy status on the IgG1/IgG2 antibody ratio to the type 1 antigen were observed, the effect of age $\times$ status approached significance $($ age $\times$ age $\times$ status, $P=0.055$ ) (Table 1, Figures $3 \mathrm{~B}, \mathrm{C}, \mathrm{D})$. Increases in IgG1 and IgG2 activity toward the type 1 antigen were observed in the majority of heifers (93\% for IgG1 and $94 \%$ for IgG2); however, the ratio of IgG1:IgG2 antibody activity toward the type 1 antigen was lower at $\mathrm{d} 14 \mathrm{P}$ compared with $\mathrm{d} 0 \mathrm{P}$ (LSM difference $=0.509$ $\pm 0.060, P<0.001)$. In contrast, IgG1 and IgG2 responses toward the type 2 antigen were observed in 80 and $57 \%$ of heifers tested, respectively, and mean IgG1 and IgG2 antibody activity toward the type 2 antigen at $\mathrm{d} 14 \mathrm{P}$ (based on raw data) was $0.34( \pm 0.05)$ and $0.06( \pm 0.01)$ OD units, respectively. Mean IgG1/IgG2 

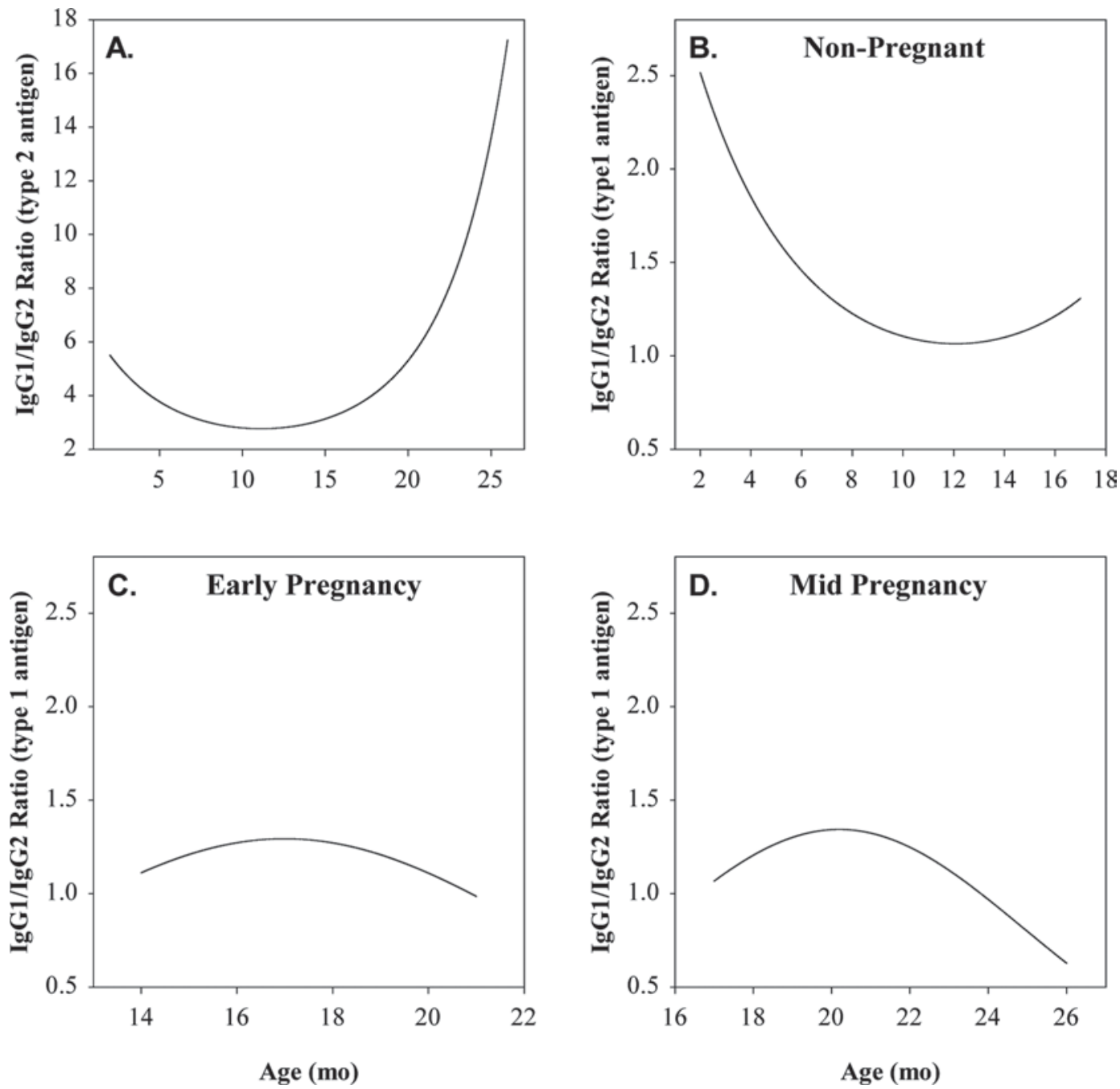

Figure 3. Effect of age on the IgG1:IgG2 antibody activity ratio against the type 2 antigen (A) and the effect of age on the IgG1:IgG2 antibody ratio against the type 1 antigen during the not pregnant (B), early pregnancy (C), and mid pregnancy (D) periods. For the IgG1:IgG2 antibody activity ratio against the type 1 antigen (but not the type 2 antigen), the effect of age varied significantly with pregnancy status.

antibody activity ratios specific to the type 1 and type 2 antigen (based on raw data) were $2.16 \pm 0.29$ and $5.90 \pm 0.49$, respectively, at d14P.

\section{Responses to Secondary IR Testing}

Positive DTH responses to the type 1 antigen were observed in all heifers that underwent secondary IR testing. Secondary DTH responses were higher in LoAMIR/HiCMIR compared with HiAMIR/LoCMIR heifers $(0.922 \pm 0.061$ vs. $0.791 \pm 0.068)$. Although not significant $(P=0.075$; Table 1$)$, a definite trend was evident in the data, suggesting that DTH responses tended to be greater in LoAMIR/HiCMIR versus HiAMIR/LoCMIR heifers. Secondary immunization led to an increase in total $\mathrm{IgG}$ antibody to the type 2 antigen at d7S relative to d0S (LSM difference $1.210 \pm 0.059$, $P<0.001)$. Positive antibody responses to the type 2 antigen were observed in all heifers that underwent secondary IR testing. Antibody to the type 2 antigen was higher in HiAMIR/LoCMIR compared with LoAMIR/ HiCMIR heifers at d7S (LSM difference $=0.389 \pm$ $0.102, P=0.003)$; however, the mean increase in total IgG antibody from d0S to d7S did not differ between phenotype classifications $(P=0.261$; Table 1$)$. The influence of phenotype classification did vary significantly depending on baseline (d0S) antibody activity $(P<0.001)$.

\section{DISCUSSION}

The ability of the putative type 1 and type 2 antigens used in IR testing to induce measurable and polarized 
CMIR and AMIR, respectively, in heifers of various ages and physiological states was confirmed, suggesting that the IR-testing protocol described in the current study can be used successfully to identify replacement heifers with enhanced overall immune responsiveness. Previous studies in dairy cattle have shown that high immune responsiveness is associated with a reduced incidence of disease, including mastitis, ketosis, and milk fever (Wagter et al., 2000; DeLaPaz, 2008; Heriazon, 2008). Heifer age and pregnancy status had significant effects on the adaptive immune response profiles of animals tested in terms of both the magnitude and type 1 and type 2 bias of responses to test antigens, highlighting the need for appropriate statistical modeling of such effects when ranking animals based on their ability to mount CMIR and AMIR.

Although several studies have investigated IR in neonatal calves and in heifers during the periparturient period, more needs to be known about the effects of age and pregnancy on IR during the period from postweaning to mid pregnancy. Passive transfer of maternal antibody protects the neonatal calf until such time as the calf's own immune system develops under the influence of environmental antigens. Although maternal antibodies are beneficial, they also can suppress antibody responses, including response to vaccines, and increase susceptibility to infection (Pastoret, 2007; Chattha et al., 2010). Immune responsiveness also is altered during the periparturient period ( 3 wk before to 3 wk after calving), in which impaired innate and acquired IR may increase incidence of disease (Mallard et al., 1998a; Wagter et al., 2000; Burton et al., 2005; Sordillo et al., 2009).

In the current study, heifer age, but not pregnancy status, influenced AMIR, as measured by total IgG specific for the type 2 antigen following primary immunization. Previous studies have demonstrated that primary and secondary antibody responses to the type 2 antigen are highly genetically correlated $(\mathrm{r}=$ 0.985; Hernández et al., 2006). In the current study, a reduced ability to mount AMIR was observed in heifers at approximately 12 mo of age. This may be a result of endocrine-immune system interactions as heifers approach sexual maturity; however, this hypothesis was not tested here. It is well recognized that the bovine endocrine and immune systems are highly integrated and do not act independently. Mallard et al. (1997) reported that serum growth hormone concentration was positively, and IGF-1 concentration negatively, correlated with antibody response in periparturient dairy cows. Treatment of dairy cows with exogenous recombinant bovine somatotropin increased serum Ig concentration (Burton et al., 1991). Results of AMIR testing in calves aged 2 to 6 mo has been reported previously (Begley et al., 2009a) and no effect of age on AMIR was observed.

Age also influenced CMIR variably, depending on pregnancy status. An age effect on DTH has been reported in calves. In newborn calves, DTH responses were significantly lower than in 21-d-old calves following priming in utero, providing evidence for suppression or incomplete maturation of CMIR in the newborn calf (Rossi et al., 1981). In dairy calves (2-6 mo), those $<3$ mo old had significantly lower DTH responses than older counterparts (Begley et al., 2009a), and, although not significant, 4- to 5-mo-old calves had lower DTH responses than calves $>5$ mo of age. Together these results suggest that CMIR in calves is enhanced with age, possibly due to a combination of immune system maturation and reduced influence of maternal antibody.

In support of these findings, results from the current study suggest that CMIR increases with age in calves from 2 to 12 mo of age and then steadily decreases. Age appears to have opposing effects on CM and AMIR. This is not surprising considering that type1/type 2 IR bias is dynamic and varies within an individual under a variety of environmental and physiological factors. Approaching parturition triggers a shift toward type 2 IR in the cow (Shafer-Weaver et al., 1999) and a negative genetic correlation exists between AMIR and CMIR in cattle (Hernández et al., 2006).

In vitro studies of bovine B cells suggest that biased type 1 and type 2 IR are linked to the production of IgG subclasses (Estes and Brown, 2002). Type 1 cytokines enhance production of IgG2 (Estes et al., 1994), whereas type 2 cytokines enhance production of IgG1, IgM and IgE (Estes et al., 1995). On this basis, the ratio of IgG1:IgG2-related antibodies produced in IR indicates type 1 or type 2 IR bias. Heifer age, but not pregnancy status, influenced the IgG1:IgG2 antibody ratio to the type 2 antigen and although not directly comparable, mean IgG1 and IgG2 antibody activity toward the type 2 antigen at d14P (based on raw data) was $0.34( \pm 0.05)$ and $0.06( \pm 0.01)$, respectively. Although there were no significant effects of age or pregnancy status on the IgG1:IgG2 antibody ratio to the type 1 antigen, the ratio of IgG1:gG2 antibody to the type 1 antigen was significantly lower at d14P as compared with d0P suggesting that as expected, antigenspecific IgG2 was predominantly produced in response to the type 1 antigen. Mean IgG1:IgG2 antibody ratios specific to the type 1 antigen were lower $(2.16 \pm 0.29)$ than for the type 2 antigen $(5.90 \pm 0.49)$. Begley et al. (2009a) reported a mean IgG1:IgG2 antibody ratio of $1.68 \pm 0.38$ to a type 2 antigen in Holstein calves. These results suggest that the putative type 1 and type 2 antigens used in the IR-testing protocol induce type 1- and type 2-biased IR, respectively, and are effective 


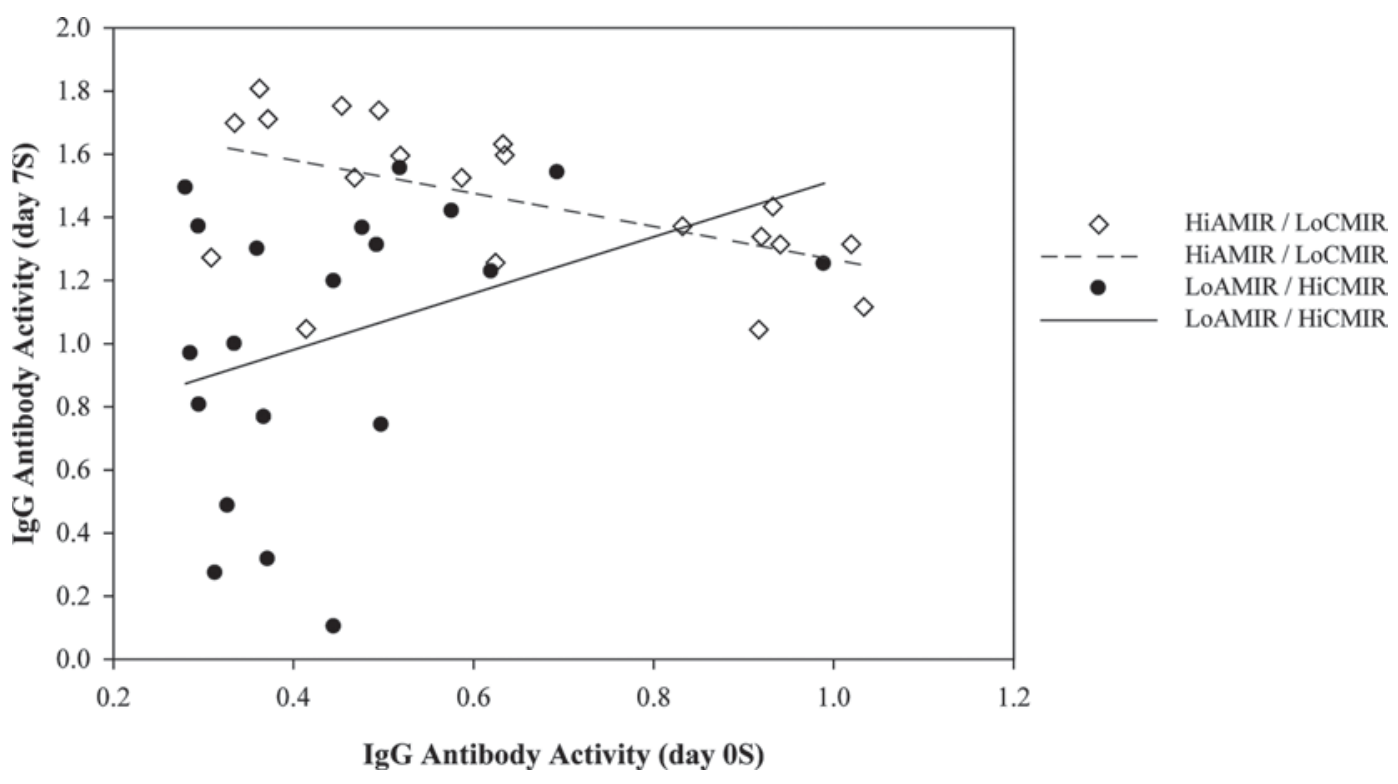

Figure 4. Relationship between total IgG antibody activity against the type 2 antigen before (d0S) and after (d7S) secondary immune response testing in heifers classified as having low (Lo)AMIR/high (Hi)CMIR or HiAMIR/LoCMIR phenotypes. Heifers were classified based on IgG antibody production specific to the type 2 antigen (AMIR = antibody-mediated immune response) and delayed-type hypersensitivity reactions to the type 1 antigen $(\mathrm{CMIR}=$ cell-mediated immune response).

test antigens to assess overall immune responsiveness. The IgG1:IgG2 antibody ratios against both the type 1 and type 2 antigen are lowest in calves at around 12 mo of age, suggesting a shift toward type 1 IR bias in calves as they approach this age. This is supported by CMIR and AMIR data indicating that DTH was high and antibody responses (both total IgG and IgG1) low at around 12 mo of age. Combined, results suggest IR in calves become increasingly type 1 biased as they approach 12 mo of age and then are increasingly type 2 biased with age and increasing gestation length.

The stability of phenotypes was evaluated by exposing a subset of animals, identified as having a HiAMIR/ LoCMIR or LoAMIR/HiCMIR phenotype following primary IR testing, to a second round of IR testing. These phenotypes were selected in the current study as they are relatively common, however, the aim of selection in a commercial setting, particularly at the outset could be to select animals which exhibit, as a minimum, an average ability to mount both a CMIR and AMIR. It is noteworthy that selection indices can be used simultaneously to make improvements in traits that are negatively correlated because a proportion of animals are high responders for both traits.

Secondary DTH responses were higher in LoAMIR/ HiCMIR versus HiAMIR/LoCMIR heifers; however, the difference only approached significance $(P=0.075)$. In contrast, antibody activity toward the type 2 antigen was higher in HiAMIR/LoCMIR compared with LoAMIR/HiCMIR heifers. However, the mean increase in antibody activity from d0S to d7S was similar for both phenotypes. When AMIR induced by secondary immunization was analyzed using a covariate model, a significant interaction of phenotype classification by OD for IgG at d0S was observed, suggesting that the relationship between antibody activity pre and post secondary immunization varies between phenotypes. A plot of OD values (raw data) for IgG at d7S versus d0S suggests that $\mathrm{d} 7 \mathrm{~S}$ and $\mathrm{d} 0 \mathrm{~S}$ values are negatively correlated in HiAMIR/LoCMIR phenotype and positively correlated in LoAMIR/HiCMIR phenotype heifers (Figure 4). Analysis estimated the difference between slopes to be $0.607 \pm 0.138$ using log OD values; however, a contribution to this observed difference by the statistical phenomenon "regression toward the mean" (Bland and Altman, 1994) must be considered.

It has been speculated that continued selection for enhanced immune responsiveness may lead to selection of animals with hyperactive immune systems and subsequently increase the incidence of autoimmune disease in selected animals. We would speculate that a threshold response level exists, and once an animal, whether of a high or low IR phenotype, reaches such a level, regulatory immune mechanisms act to dampen continued response. This suggests that high-responder phenotypes that exhibit enhanced responses to antigens require only limited exposure to reach a level of IR that is protective, whereas low-responder phenotypes require multiple or extended exposure to antigen to reach the same level of response. The ability of high-responder 
phenotype animals to reach these levels more rapidly than their low-responder counterparts allows them to downregulate IR upon continued antigen exposure, thereby minimizing the biological costs of continual immune system stimulation and reducing detrimental effects of inflammation (Colditz, 2008). On this basis, combining results of primary and secondary IR testing may disadvantage HiAMIR phenotype animals by underestimating their ability to mount an AMIR.

Selection for overall immune responsiveness has been investigated in pigs (Mallard et al., 1998b; Wilkie and Mallard, 1999). Pigs, classified as high, average, or low immune responders using a combination of EBV for CMIR and AMIR were line-bred for a total of 8 generations and the progeny tested for resistance to a variety of infectious diseases. High-responder pigs had lower overall clinical scores to Mycoplasma hyorhinis infection, although they had significantly more severe arthritis (Magnusson et al., 1998; Jayagopala Reddy et al., 2000). Additionally, antibody responses to a variety of antigens were significantly enhanced (both in quantity and avidity) in high-responder pigs compared with their low-responder counterparts (Appleyard et al., 1992; Mallard et al., 1998b). High-responder pigs also had higher rates of weight gain, suggesting that selection for immune responsiveness may lead to gains in productivity.

Based on heritability estimates of 0.41 and 0.37 for bovine CMIR and AMIR, respectively (Hernández et al., 2006), moderate to high genetic gains can be expected when selecting for overall immune responsiveness, making this a feasible genetic approach to reducing the incidence of disease. In contrast, heritability estimates for the majority of health traits relating to an individual disease are low to moderate (Lyons et al., 1991; Uribe et al., 1995). For example, the heritability of clinical mastitis incidence has been estimated at 0.03 in Norwegian Red cows and between 0 and 0.15 (depending on lactation number) in Holstein cows (Lyons et al., 1991; Uribe et al., 1995; Van Dorp et al., 1998). Although breeding for resistance to individual diseases has merit in certain circumstances, selection for resistance to a specific disease may inadvertently increase susceptibility to other diseases. Highlighting the risks of selection for resistance to individual diseases, Price et al. (1990) reported an inverse relationship between antibody production and macrophage function in cattle selected for resistance or susceptibility to Brucella abortus. A similar relationship also has been reported in mice selected for high and low antibody production (Biozzi et al., 1984, Hale and Howard, 1981), whereas in pigs selected for overall immune responsiveness, monocyte function was similar in high-IR (HiAMIR/HiCMIR) and lowIR (LoAMIR/LoCMIR) lines (Groves et al., 1993).
Future studies will be aimed at estimating genetic correlations between overall immune responsiveness and disease-resistance traits in Canadian dairy herds by selecting cows based on overall immune responsiveness over several generations and testing their resistance to a variety of infectious diseases. A comparison of disease resistance in selected versus random bred animals is expected to highlight the benefits of selection based on overall immune responsiveness.

We describe here the successful use of a patented protocol to assess overall immune responsiveness in replacement heifers, and demonstrate the effects of age and pregnancy status on type 1 and type 2 IR in Canadian Holstein heifers. An understanding of the effects of age and pregnancy on type 1 and type 2 IR is critical to the accuracy of predicting an animal's overall immune responsiveness. Knowledge of the influences of age and pregnancy on the dynamics of type 1 and type 2 IR bias in heifers also may aid in the development of effective vaccines through strategic targeting of vaccine components to recipients.

\section{ACKNOWLEDGMENTS}

The authors acknowledge the financial support provided by the Canadian Bovine Mastitis Research Network. Special thanks also are extended to L. Wright and the staff at the University of Guelph's Elora Dairy research facility for their cooperation and assistance; to L. Pyne, A. Murdoch, and J. Galliene for providing technical assistance, W. Sears for statistical advice, and B. Wilkie (all of the University of Guelph, Ontario, Canada) for valuable discussions.

\section{REFERENCES}

Appleyard, G., B. N. Wilkie, B. W. Kennedy, and B. A. Mallard. 1992. Antibody avidity in Yorkshire pigs of high and low immune response groups. Vet. Immunol. Immunopathol. 31:229-240.

Begley, N., F. Buckley, E. B. Burnside, L. Schaeffer, K. Pierce, and B. A. Mallard. 2009a. Immune responses of Holstein and Norwegian Red $\times$ Holstein calves on Canadian dairy farms. J. Dairy Sci. 92:518-525.

Begley, N., F. Buckley, K. M. Pierce, A. G. Fahey, and B. A. Mallard. 2009b. Differences in udder health and immune response traits of Holstein-Friesians, Norwegian Reds, and their crosses in second lactation. J. Dairy Sci. 92:749-757.

Biozzi, G., D. Mouton, C. Stiffel, and Y. Bouthillier. 1984. A major role of the macrophage in quantitative genetic regulation of immunoresponsiveness and antiinfectious immunity. Adv. Immunol. $36: 189-234$.

Bland, J. M., and D. G. Altman. 1994. Regression towards the mean. BMJ 308:1499.

Burton, J. L., S. A. Madsen, L. C. Chang, P. S. Weber, K. R. Buckham, R. van Dorp, M. C. Hickey, and B. Earley. 2005. Gene expression signatures in neutrophils exposed to glucocorticoids: A new paradigm to help explain "neutrophil dysfunction" in parturient dairy cows. Vet. Immunol. Immunopathol. 105:197-219.

Burton, J. L., B. W. McBride, B. W. Kennedy, J. H. Burton, T. H. Elsasser, and B. Woodward. 1991. Serum immunoglobulin profiles 
of dairy cows chronically treated with recombinant bovine somatotropin. J. Dairy Sci. 74:1589-1598.

Chattha, K. S., M. A. Firth, D. C. Hodgins, and P. E. Shewen. 2010. Variation in expression of membrane IgM, CD21 (CR2) and CD32 (Fcgamma RIIB) on bovine lymphocytes with age: A longitudinal study. Dev. Comp. Immunol. 34:510-517.

Colditz, I. G. 2008. Six costs of immunity to gastrointestinal nematode infections. Parasite Immunol. 30:63-70.

Crawley, A., and B. N. Wilkie. 2003. Porcine Ig isotypes: Function and molecular characteristics. Vaccine 21:2911-2922.

Crawley, A. M., B. Mallard, and B. N. Wilkie. 2005. Genetic selection for high and low immune response in pigs: Effects on immunoglobulin isotype expression. Vet. Immunol. Immunopathol. 108:71-76.

DeLaPaz, J. 2008. Antibody and cell-mediated immune responses to novel antigens in periparturient dairy cows as a measure of disease resistance. MSc Thesis. College of Vet. Med., Univ. of Florida, Gainesville.

Estes, D. M., and W. C. Brown. 2002. Type 1 and type 2 responses in regulation of Ig isotype expression in cattle. Vet. Immunol. Immunopathol. 90:1-10.

Estes, D. M., N. M. Closser, and G. K. Allen. 1994. IFN-gamma stimulates IgG2 production from bovine B cells costimulated with antimu and mitogen. Cell. Immunol. 154:287-295.

Estes, D. M., A. Hirano, V. T. Heussler, D. A. Dobbelaere, and W. C. Brown. 1995. Expression and biological activities of bovine interleukin 4: Effects of recombinant bovine interleukin 4 on $\mathrm{T}$ cell proliferation and $\mathrm{B}$ cell differentiation and proliferation in vitro. Cell. Immunol. 163:268-279.

Groves, T. C., B. N. Wilkie, B. W. Kennedy, and B. A. Mallard. 1993. Effect of selection of swine for high and low immune responsiveness on monocyte superoxide anion production and class II MHC antigen expression. Vet. Immunol. Immunopathol. 36:347-358.

Hale, C., and J. G. Howard. 1981. Immunological regulation of experimental cutaneous leishmaniasis. 2. Studies with Biozzi high and low responder lines of mice. Parasite Immunol. 3:45-55.

Heriazon, A. 2008. Phenotypic and genetic parameters of acquired immune response to improve dairy cattle health. PhD Thesis. Univ. of Guelph, ON, Canada.

Heriazon, A., K. A. Thompson, B. N. Wilkie, W. Mathes-Sears, M. Quinton, and B. A. Mallard. 2009a. Antibody to ovalbumin and delayed-type hypersensitivity to Candida albicans and mycobacteria in lactating Holstein cows using Quil A or Freund's complete adjuvant. Vet. Immunol. Immunopathol. 127:220-227.

Heriazon, A., J. A. Yager, W. Sears, and B. A. Mallard. 2009b. Induction of delayed-type hypersensitivity and interferon-gamma to Candida albicans and anti-hen-egg white lysozyme antibody as phenotypic markers of enhanced bovine immune response. Vet. Immunol. Immunopathol. 129:93-100.

Hernández, A., N. Karrow, and B. A. Mallard. 2003. Evaluation of immune responses of cattle as a means to identify high or low responders and use of a human microarray to differentiate gene expression. Genet. Sel. Evol. 35(Suppl. 1):S67-S81.

Hernández, A., V. M. Quinton, F. Miglior, and B. A. Mallard. 2006. Genetic parameters of dairy cattle immune response traits. Proc. 8th World Congr. Genet. Appl. Livest. Prod., Belo Horizonte, Brazil.

Hernández, A., J. A. Yager, B. N. Wilkie, K. E. Leslie, and B. A. Mallard. 2005. Evaluation of bovine cutaneous delayed-type hypersensitivity (DTH) to various test antigens and a mitogen using several adjuvants. Vet. Immunol. Immunopathol. 104:45-58.

Jayagopala Reddy, N. R., B. N. Wilkie, P. Borgs, and B. A. Mallard. 2000. Cytokines in mycoplasma hyorhinis-induced arthritis in pigs bred selectively for high and low immune responses. Infect. Immun. 68:1150-1155.
Lyons, D. T., A. E. Freeman, and A. L. Kuck. 1991. Genetics of health traits in Holstein cattle. J. Dairy Sci. 74:1092-1100.

Magnusson, U., B. Wilkie, B. Mallard, S. Rosendal, and B. Kennedy. 1998. Mycoplasma hyorhinis infection of pigs selectively bred for high and low immune response. Vet. Immunol. Immunopathol. 61:83-96.

Mallard, B. A., and L. Wagter. 2001. Method of identifying high immune response animals. University of Guelph, Ontario, Canada, assignee. US Pat. No. 6,287,564.

Mallard, B. A., J. C. Dekkers, M. J. Ireland, K. E. Leslie, S. Sharif, C. L. Vankampen, L. Wagter, and B. N. Wilkie. 1998a. Alteration in immune responsiveness during the peripartum period and its ramification on dairy cow and calf health. J. Dairy Sci. 81:585-595.

Mallard, B. A., L. C. Wagter, M. J. Ireland, and J. C. Dekkers. 1997. Effects of growth hormone, insulin-like growth factor-I, and cortisol on periparturient antibody response profiles of dairy cattle. Vet. Immunol. Immunopathol. 60:61-76.

Mallard, B. A., B. N. Wilkie, B. W. Kennedy, J. Gibson, and M. Quinton. 1998b. Immune responsiveness in swine: Eight generations of selection for high and low immune response in Yorkshire pigs. Pages 257-264 in Proc. 6th World Congr. Genet. Appl. Livest. Prod., Armidale, NSW, Australia.

Nagahata, H., A. Ogawa, Y. Sanada, H. Noda, and S. Yamamoto. 1992. Peripartum changes in antibody producing capability of lymphocytes from dairy cows. Vet. Q. 14:39-40.

Nino-Soto, M. I., R. J. Jozani, B. Bridle, and B. A. Mallard. 2008. CDNA microarray analysis of gene expression patterns in blood mononuclear cells of SLA-DRB1-defined Yorkshire pigs. Dev. Biol. (Basel) 132:321-325.

Pastoret, P. P. 2007. Challenges and issues of early life vaccination in animals and humans. J. Comp. Pathol. 137(Suppl. 1):S2-S3

Price, R. E., J. W. Templeton, R. Smith 3rd, and L. G. Adams. 1990. Ability of mononuclear phagocytes from cattle naturally resistant or susceptible to brucellosis to control in vitro intracellular survival of Brucella abortus. Infect. Immun. 58:879-886.

Rossi, C. R. G. K. Kiesel, R. S. Hudson, T. A. Powe, and L. F. Fisher 1981. Evidence for suppression or incomplete maturation of cellmediated immunity in neonatal calves as determined by delayedtype hypersensitivity responses. Am. J. Vet. Res. 42:1369-1370.

Saad, A. M., C. Concha, and G. Astrom. 1989. Alterations in neutrophil phagocytosis and lymphocyte blastogenesis in dairy cows around parturition. Zentralbl. Veterinarmed. B. 36:337-345.

Shafer-Weaver, K. A., C. M. Corl, and L. M. Sordillo. 1999. Shifts in bovine CD4+ subpopulations increase T-helper-2 compared with T-helper-1 effector cells during the postpartum period. J. Dairy Sci. 82:1696-1706.

Sordillo, L. M., G. A. Contreras, and S. L. Aitken. 2009. Metabolic factors affecting the inflammatory response of periparturient dairy cows. Anim. Health Res. Rev. 10:53-63.

Uribe, H. A., B. W. Kennedy, S. W. Martin, and D. F. Kelton. 1995. Genetic parameters for common health disorders of Holstein cows. J. Dairy Sci. 78:421-430.

Van Dorp, T. E., J. C. Dekkers, S. W. Martin, and J. P. Noordhuizen. 1998. Genetic parameters of health disorders, and relationships with 305-day milk yield and conformation traits of registered Holstein cows. J. Dairy Sci. 81:2264-2270.

Wagter, L. C., B. A. Mallard, B. N. Wilkie, K. E. Leslie, P. J. Boettcher, and J. C. Dekkers. 2000. A quantitative approach to classifying Holstein cows based on antibody responsiveness and its relationship to peripartum mastitis occurrence. J. Dairy Sci. 83:488-498.

Wilkie, B., and B. Mallard. 1999. Selection for high immune response: An alternative approach to animal health maintenance? Vet. Immunol. Immunopathol. 72:231-235. 